\title{
Parametric Macromodeling of Lossy and Dispersive Multiconductor Transmission Lines
}

\author{
Francesco Ferranti, Giulio Antonini, Senior Member, IEEE, Tom Dhaene, Senior Member, IEEE, and \\ Luc Knockaert, Senior Member, IEEE
}

\begin{abstract}
We propose an innovative parametric macromodeling technique for lossy and dispersive multiconductor transmission lines (MTLs) that can be used for interconnect modeling. It is based on a recently developed method for the analysis of lossy and dispersive MTLs extended by using the Multivariate Orthonormal Vector Fitting (MOVF) technique to build parametric macromodels in a rational form. They take into account design parameters, such as geometrical layout or substrate features, in addition to frequency. The presented technique is suited to generate state-space models and synthesize equivalent circuits, which can be easily embedded into conventional SPICElike solvers. Parametric macromodels allow to perform design space exploration, design optimization, and sensitivity analysis efficiently. Numerical examples validate the proposed approach in both frequency and time domain.
\end{abstract}

Index Terms-Interconnects, parametric macromodeling, rational approximation, transient analysis.

\section{INTRODUCTION}

The increasing demand for performance of integrated circuits (ICs) pushes operation to higher signal bandwidths, while rapid advances in manufacturing capabilities have significantly reduced the feature size and density of these devices. In order to assist microwave designers, accurate modeling of previously neglected second order effects, such as crosstalk, reflection, delay and coupling, becomes increasingly important during circuit and system simulations. The accurate prediction of these interconnects effects is fundamental for a successful design and involves the solution of large systems of equations which are often prohibitively CPU expensive to solve [1]- [2]. Various levels of design hierarchy, such as on-chip, packaging and printed circuit boards, require an accurate and efficient macromodeling of interconnects effects. Over the years, many macromodeling techniques have been developed [3]- [26].

More recently, a spectral approach has been presented for the analysis of lossy and dispersive multiconductor transmission lines [27]. It is based on the computation of the closedform dyadic Green's function of the 1-D wave propagation

Manuscript received December 2008.

Francesco Ferranti, Tom Dhaene and Luc Knockaert are with the Department of Information Technology (INTEC), at Ghent University - IBBT, Sint Pietersnieuwstraat 41, 9000 Ghent, Belgium, email: francesco.ferranti@intec.ugent.be; tom.dhaene@intec.ugent.be; luc.knockaert@intec.ugent.be.

Giulio Antonini is with the UAq EMC Laboratory, Dipartimento di Ingegneria Elettrica e dell'Informazione, Università degli Studi dell'Aquila, Monteluco di Roio, 67040, L'Aquila, Italy, phone: +390862434462, e-mail: antonini@ing.univaq.it.

This work was supported by the Research Foundation Flanders (FWO) and by the Italian Ministry of University (MIUR) under a Program for the Development of Research of National Interest, (PRIN grant n. 2006095890). problem. The propagation of voltage along a multiconductor transmission line is described by a second order differential equations which is found to be a self-adjoint problem [28]. As a consequence, the corresponding Green's function can be expanded in a series form of orthonormal basis functions which is well suited for poles and residues identification and, thus, for time-domain macromodeling purposes. The major advantage of such an approach over existing techniques consists of the rational nature of the dyadic Green's function which is appropriate for time-domain macromodeling. Furthermore, the use of orthonormal basis functions to expand the solution allows to compute the poles and residues of the system independently for each mode, and this reduce the complexity of the system identification significantly.

Parametric macromodels are important for design space exploration, design optimization, and sensitivity analysis. For example, once the fabrication technology is decided, an optimization step is required at the early design stages to select the geometrical and material features of the structure, such as length, height and width of conductors, dielectric permittivity and metal conductivity, yielding the optimum electrical performance, often under stringent signal integrity and electromagnetic compatibility constraints. To make efficient and feasible these design activities, parametric macromodeling techniques that take into account design parameters in addition to frequency (or time) are needed. Their realization by using full electromagnetic simulations on the entire parameter space is often computationally expensive. Some techniques for parametric macromodeling of MTLs were proposed in the framework of model order reduction [29]- [30]. Recently, another parametrization scheme based on the generalized method of characteristics (MoC) was presented in [31]. We developed a new parametric macromodeling technique, presented in this paper, with the aim of realizing the previously cited design activities efficiently, reducing the computational resources required by extensive electromagnetic simulations. It is based on the spectral approach presented in [27] for lossy and dispersive MTLs, coupled with the Multivariate Orthonormal Vector Fitting (MOVF) technique [32] to handle other design parameters in addition to frequency. MOVF permits to build rational parametric macromodels starting from multivariate data samples in the parameter space and combines the use of an iterative least squares estimator and orthonormal rational functions, which are based on a prescribed set of poles. Based on a fixed set of design parameters, the multivariate model can easily be reduced to a univariate frequency-dependent model in a rational form, that is suitable to generate a finite state- 
space representation and an equivalent SPICE circuit by using standard realization [33] and circuit synthesis techniques [34].

This paper is structured as follows. First, an overview of the spectral approach for MTLs and MOVF technique is given in Sections II and III. Then, Section IV explains how both the methods are coupled to build a parametric representation of a MTL system, presenting different possible flavors. Finally, some numerical examples are presented in Sections V, validating the proposed technique.

\section{Spectral Modeling of Multiconductor TRANSMISSION LINES}

Multiconductor transmission lines are described by the following set of partial differential equations, known as Telegrapher's equations, which, at the generic abscissa $z$, in the Laplace domain, read [35]:

$$
\begin{aligned}
\frac{d}{d z} \boldsymbol{V}(z, s) & =-\left[\boldsymbol{R}_{p u l}(s)+s \boldsymbol{L}_{p u l}(s)\right] \boldsymbol{I}(z, s) \\
& =-\boldsymbol{Z}_{p u l}(s) \boldsymbol{I}(z, s) \\
\frac{d}{d z} \boldsymbol{I}(z, s) & =-\left[\boldsymbol{G}_{p u l}(s)+s \boldsymbol{C}_{p u l}(s)\right] \boldsymbol{V}(z, s)+\boldsymbol{I}_{S}(z, s) \\
& =-\boldsymbol{Y}_{p u l}(s) \boldsymbol{V}(z, s)+\boldsymbol{I}_{S}(z, s) .
\end{aligned}
$$

where $\boldsymbol{R}_{\text {pul }}(s), \boldsymbol{L}_{p u l}(s), \boldsymbol{C}_{\text {pul }}(s)$ and $\boldsymbol{G}_{p u l}(s)$ are frequencydependent per-unit-length parameter matrices and are nonnegative definite symmetric matrices of order $N, N+1$ being the number of the conductors [35], [36]; $\boldsymbol{I}_{S}(z, s)$ represents a per-unit-length current source located at abscissa $z$, which, since we assume that currents are injected into the system only at abscissas $z=0, z=\ell$, is given by

$$
\boldsymbol{I}_{S}(z, s)=\boldsymbol{I}_{0}(s) \delta(z)+\boldsymbol{I}_{\ell}(s) \delta(z-\ell)
$$

Vectors $\boldsymbol{V}(z, s)$ and $\boldsymbol{I}(z, s)$ represent the voltage and current vectors depending on Laplace variable $s$ and position $z$ along the line.

Some trivial manipulations of (1) leads to

$$
\frac{d^{2}}{d z^{2}} \boldsymbol{V}(z, s)-\gamma^{2}(s) \boldsymbol{V}(z, s)=-\boldsymbol{Z}_{p u l}(s) \boldsymbol{I}_{S}(z, s)
$$

where $\gamma^{2}(s)=\boldsymbol{Z}_{\text {pul }}(s) \boldsymbol{Y}_{p u l}(s)$. Since the port currents are treated as per-unit-length sources, homogeneous boundary conditions of the Neumann type can be adopted for the voltage satisfying (3)

$$
\left.\frac{d}{d z} \boldsymbol{V}(z, s)\right|_{z=0}=\left.\frac{d}{d z} \boldsymbol{V}(z, s)\right|_{z=\ell}=\mathbf{0}
$$

The differential system of equations (3) with boundary conditions problem (4) can be regarded as a Sturm-Liouville problem with boundary conditions of the Neumann type. The general solution for the voltage at abscissa $z$ of the multiconductor transmission line due to the port currents is obtained in [27] and briefly reported here for completeness:

$$
\begin{aligned}
\boldsymbol{V}(z, s) & =\int_{0}^{\ell} \underline{\boldsymbol{G}}_{V}\left(z, z^{\prime}, s\right)\left(-\boldsymbol{Z}_{p u l}(s) \boldsymbol{I}_{S}\left(z^{\prime}, s\right)\right) d z^{\prime} \\
& =\underline{\boldsymbol{G}}_{V}(z, 0, s)\left(-\boldsymbol{Z}_{p u l}(s) \boldsymbol{I}(0, s)\right)+ \\
& +\underline{\boldsymbol{G}}_{V}(z, \ell, s)\left(-\boldsymbol{Z}_{p u l}(s) \boldsymbol{I}(\ell, s)\right) .
\end{aligned}
$$

In [27] it has been found that the dyadic Green's function $\underline{\boldsymbol{G}}_{V}\left(z, z^{\prime}, s\right)$ for the multiconductor transmission line problem can be written as:

$$
\underline{\boldsymbol{G}}_{V}\left(z, z^{\prime}, s\right)=-\sum_{n=0}^{\infty} \phi_{n}(s) A_{n}^{2} \psi_{n}(z) \psi_{n}\left(z^{\prime}\right),
$$

where

$$
\begin{aligned}
& \phi_{n}(s)=\left[\gamma^{2}(s)+\left(\frac{n \pi}{\ell}\right)^{2} \boldsymbol{U}\right]^{-1}, \\
& \psi_{n}(z)=\cos \left(\frac{n \pi}{\ell} z\right),
\end{aligned}
$$

and $A_{0}=\sqrt{1 / \ell}, A_{n}=\sqrt{2 / \ell}, n=1, \cdots, \infty, \boldsymbol{U}$ is the unitary dyadic. Finally, the spectral representation of the $Z$ impedance matrix is generated as:

$$
\begin{aligned}
{\left[\begin{array}{l}
\boldsymbol{V}(0, s) \\
\boldsymbol{V}(\ell, s)
\end{array}\right] } & =\left[\begin{array}{ll}
\boldsymbol{Z}_{11} & \boldsymbol{Z}_{12} \\
\boldsymbol{Z}_{21} & \boldsymbol{Z}_{22}
\end{array}\right] \cdot\left[\begin{array}{c}
\boldsymbol{I}(0, s) \\
\boldsymbol{I}(\ell, s)
\end{array}\right] \\
& =\sum_{n=0}^{\infty}\left[\begin{array}{ll}
\boldsymbol{Z}_{n, 11} & \boldsymbol{Z}_{n, 12} \\
\boldsymbol{Z}_{n, 21} & \boldsymbol{Z}_{n, 22}
\end{array}\right] \cdot\left[\begin{array}{c}
\boldsymbol{I}(0, s) \\
\boldsymbol{I}(\ell, s)
\end{array}\right],
\end{aligned}
$$

where

$$
\begin{aligned}
\boldsymbol{Z}_{11} & =\boldsymbol{Z}_{22} \\
& =\sum_{n=0}^{\infty}\left[\gamma^{2}(s)+\left(\frac{n \pi}{\ell}\right)^{2} \boldsymbol{U}\right]^{-1} \cdot A_{n}^{2} \boldsymbol{Z}_{p u l}(s), \\
\boldsymbol{Z}_{12} & =\boldsymbol{Z}_{21} \\
& =\sum_{n=0}^{\infty}\left[\gamma^{2}(s)+\left(\frac{n \pi}{\ell}\right)^{2} \boldsymbol{U}\right]^{-1} \cdot A_{n}^{2} \boldsymbol{Z}_{p u l}(s)(-1)^{n}
\end{aligned}
$$

It is composed of an infinite number of modes $Z_{n}$. The poles of (9) are those of the Green's function (6) which can be calculated as in [27]. A rational form can be obtained for (9) by computing the corresponding residues by standard techniques [37]. The series form of the dyadic Green's function is very general; it assumes that the multiconductor transmission line supports the quasi-TEM mode and is uniform along the $z$-axis. No hypothesis has been done regarding the nature of the perunit-length longitudinal impedance $\boldsymbol{Z}_{p u l}(s)$ and transversal admittance $\boldsymbol{Y}_{\text {pul }}(s)$ matrix and, as a consequence, on the propagation constant $\gamma^{2}(s)$. Thus, the proposed model can be used for transmission lines with either frequency-independent or frequency-dependent per-unit-length parameters [27]. This means that skin-effect and dielectric polarization losses can be easily modeled and incorporated in transient analysis once the frequency-dependent per-unit-length parameters are available.

\section{Multivariate Orthonormal Vector FitTing TECHNIQUE}

This section presents an overview of MOVF technique that permits to build parametric macromodels, taking into account other design parameters, such as geometrical layout or substrate features, in addition to frequency. For ease of notation, MOVF algorithm is only described for bivariate systems. Of course, the full multivariate formulation can be derived in a similar way. It proposes to represent the 
parametric macromodel as the ratio of a bivariate numerator and denominator

$$
\boldsymbol{F}(s, g)=\frac{\boldsymbol{N}(s, g)}{D(s, g)}=\frac{\sum_{p=0}^{P} \sum_{v=0}^{V} \boldsymbol{c}_{p v} \phi_{p}(s) \varphi_{v}(g)}{\sum_{p=0}^{P} \sum_{v=0}^{V} \tilde{c}_{p v} \phi_{p}(s) \varphi_{v}(g)}
$$

where $s$ is the complex frequency variable and $g$ is a real design variable. The maximum order of the corresponding basis functions $\phi_{p}(s)$ and $\varphi_{v}(g)$ is denoted by $P$ and $V$ respectively. Based on a set of data samples $\left\{(s, g)_{k}, H(s, g)_{k}\right\}_{k=1}^{K}$, the algorithm pursues the identification of the model coefficients $c_{p v}$ and $\tilde{c}_{p v}$ of numerator and denominator in (10). A linear approximation to this nonlinear optimization problem is obtained by using an iterative procedure explained in the next section. In this work the MOVF technique is applied to matrices and it is assumed that the different matrix entries share the same poles, so the same denominator $D(s, g)$. In (10) the number of coefficients $\boldsymbol{c}_{p v}$ is equal to $(P+1) \cdot(V+1) \cdot M$ where $M$ is the maximum number of functions fitted with common poles in the same least-squares matrix. The number of coefficients $\tilde{c}_{p v}$ is equal to $(P+1) \cdot(V+1)$, the denominator term is the same for all the functions fitted using common poles. Increasing the number of ports and poles required for the fitting, the memory requirement to obtain the model by MOVF can be high, for this reason the authors advise to use the first two parametric macromodeling strategies described in Sections IV.A. and IV.B, which exploit the modal decomposition to reduce the complexity of the modeling process significantly.

\section{A. Iterative Algorithm}

In the first iteration step of the algorithm $(t=0)$, Levi's cost function [38] is minimized to obtain an initial guess of the coefficients. In successive iteration steps $(t=1, \ldots, T)$, the Sanathanan-Koerner (SK) cost function is minimized [39], which uses the inverse of the previously estimated denominator

$$
\left(D^{(t-1)}(s, g)_{k}\right)^{-1}=w^{(t)}(s, g)_{k}
$$

as an explicit weight factor to the least-squares equations. A relaxed non-triviality constraint is added as an additional row in the system matrix [32], to avoid the trivial null solution and improve the convergence of the algorithm. Each equation is split in its real and imaginary parts, to ensure that the model coefficients $\boldsymbol{c}_{p v}^{(t)}, \tilde{c}_{p v}^{(t)}$ are real. Scaling each column to unity length [40] is suitable to improve the numerical accuracy of the results.

\section{B. Choice of basis functions}

In this section the choice of the basis functions for the complex frequency variable $s$ and the real design variable $g$ is presented.

1) Frequency-dependent basis functions: Based on a prescribed set of stable poles $\boldsymbol{a}=\left\{-a_{p}\right\}_{p=1}^{P}$, a set of partial fractions $\phi_{p}(s, \boldsymbol{a})$ is chosen, with $\phi_{0}(s)=1$. These poles are grouped as complex conjugate pole pairs, and are selected such that they have small negative real parts and the imaginary parts linearly spaced over the frequency range of interest [40]. In order to make the transfer function coefficients real-valued, a linear combination of $\phi_{p}(s, \boldsymbol{a})$ and $\phi_{p+1}(s, \boldsymbol{a})$ is formed as follows

$$
\begin{aligned}
\phi_{p}(s, \boldsymbol{a}) & =\left(s+a_{p}\right)^{-1}+\left(s+a_{p+1}\right)^{-1} \\
\phi_{p+1}(s, \boldsymbol{a}) & =j\left(s+a_{p}\right)^{-1}-j\left(s+a_{p+1}\right)^{-1}
\end{aligned}
$$

To improve the numerical stability of the modeling algorithm, a set of orthonormal basis functions can be used, as shown in [41]. The orthonormal basis functions can improve the conditioning of the system equations and are less sensitive to the choice of the initial poles.

2) Parameter-dependent basis functions: The parameterdependent basis functions $\varphi_{v}(g, \boldsymbol{b})$ are also chosen in partial fraction form as a function of $j g$, hence in rational form. The starting poles of $\varphi_{v}(g, \boldsymbol{b})$ and $\varphi_{v+1}(g, \boldsymbol{b})$ are chosen as complex pairs $b_{v+1}=-\left(b_{v}\right)^{*}$ which have small real parts of opposite sign $\left(-\alpha_{v}, \alpha_{v}\right)$, and their imaginary parts $\beta_{v}$ linearly spaced over the parameter range of interest, such that

$$
\begin{gathered}
-b_{v}=-\alpha_{v}+j \beta_{v},-b_{v+1}=\alpha_{v}+j \beta_{v} \\
\left\{\alpha_{v}\right\}=0.01\left\{\beta_{v}\right\}
\end{gathered}
$$

while $\varphi_{0}(g)=1$. A linear combination of two fractions is used to ensure that $\varphi_{v}(g, \boldsymbol{b})$ and $\varphi_{v+1}(g, \boldsymbol{b})$ are real functions [32]:

$$
\begin{array}{r}
\varphi_{v}(g, \boldsymbol{b})=\left(j g+b_{v}\right)^{-1}-\left(j g-\left(b_{v}\right)^{*}\right)^{-1} \\
\varphi_{v+1}(g, \boldsymbol{b})=j\left(j g+b_{v}\right)^{-1}+j\left(j g-\left(b_{v}\right)^{*}\right)^{-1}
\end{array}
$$

\section{Additional weighting function}

An additional least-squares weighting function can be added to the parametric macromodeling algorithm, when the elements to fit have a high dynamic range. It improves the relative accuracy where the elements to fit are small in their dynamic range [42] and is chosen equal to the inverse of the element magnitude:

$$
w_{H_{i}}(s, g)_{k}=\left|\left(H_{i}(s, g)_{k}\right)^{-1}\right|
$$

for $i=1, \ldots, M$. The RMS-error is chosen to characterize the model accuracy. It is weighted if the previous weighting function is used during the modeling process.

$$
R M S=\sqrt{\frac{1}{M K} \sum_{i=1}^{M} \sum_{k=1}^{K}\left|R_{i}(s, g)_{k}-H_{i}(s, g)_{k}\right|^{2}}
$$

$$
\begin{aligned}
& R M S_{\text {weighted }}= \\
& =\sqrt{\frac{1}{M K} \sum_{i=1}^{M} \sum_{k=1}^{K}\left|w_{H_{i}}(s, g)_{k}\left(R_{i}(s, g)_{k}-H_{i}(s, g)_{k}\right)\right|^{2}}
\end{aligned}
$$

\section{Parametric Macromodeling Strategies}

In this section, we extend the spectral MTL modeling approach coupling it with MOVF technique, to be able to generate MTL parametric representation. Three different parametric macromodeling strategies are presented. 
A. Parametric macromodeling of $\boldsymbol{Z}_{\text {pul }}(s, g)$ and $\boldsymbol{Y}_{\text {pul }}(s, g)$

The per-unit-length impedance and admittance $\boldsymbol{Z}_{p u l}(s, g)$ and $\boldsymbol{Y}_{p u l}(s, g)$ are modeled as functions of the frequency and other design parameters. The length is not a parameter for this approach.

$$
\begin{aligned}
& \boldsymbol{Z}_{p u l}(s, g) \simeq \tilde{\boldsymbol{Z}}_{p u l}(s, g)=\frac{\boldsymbol{N}_{Z_{p u l}}(s, g)}{D_{Z_{p u l}}(s, g)}
\end{aligned}
$$

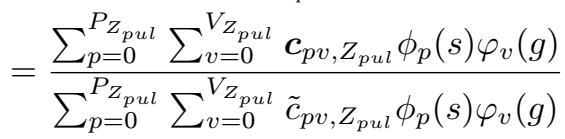

$$
\begin{aligned}
& \boldsymbol{Y}_{p u l}(s, g) \simeq \tilde{\boldsymbol{Y}}_{p u l}(s, g)=\frac{\boldsymbol{N}_{Y_{p u l}}(s, g)}{D_{Y_{p u l}}(s, g)} \\
& =\frac{\sum_{p=0}^{P_{Y_{p u l}}} \sum_{v=0}^{V_{Y_{p u l}}} \boldsymbol{c}_{p v, Y_{p u l}} \phi_{p}(s) \varphi_{v}(g)}{\sum_{p=0}^{P_{Y_{p u l}}} \sum_{v=0}^{V_{Y_{p u l}}} \tilde{c}_{p v, Y_{p u l}} \phi_{p}(s) \varphi_{v}(g)}
\end{aligned}
$$

Once these per-unit-length parametric macromodels are built, given a fixed set of values for the parameters, they can be reduced to univariate frequency-dependent functions as in [32]. Since MOVF does not guarantee stability and passivity of the parametric macromodel by construction, the stability of the univariate model can be imposed in the reduction step using pole flipping, and, subsequently, passivity can be enforced in a post-processing step by means of standard techniques (see [43] and [44]).

After these steps, a univariate rational model is obtained for the $Z$ matrix as shown in [27]. At this stage, the length of the MTL system is chosen. This rational model is passive and stable, if passivity and stability are imposed on the univariate models of the per-unit-length impedance and admittance [27]. Finally, a state space representation and an equivalent SPICE circuit can be realized for the $Z$ matrix, by using standard realization [33] and circuit synthesis techniques [34].

\section{B. Parametric macromodeling of modal impedances $\boldsymbol{Z}_{n}(s, g)$}

The spectral approach for multiconductor transmission lines allows to decompose the impedance matrix entries in modal impedances which have a rational form as shown in (9). They can be modeled by a multivariate representation. In this approach the length is also seen as a design parameter.

$$
\begin{aligned}
& \boldsymbol{Z}_{n}(s, g) \simeq \tilde{\boldsymbol{Z}}_{n}(s, g)=\frac{\boldsymbol{N}_{Z_{n}}(s, g)}{D_{Z_{n}}(s, g)} \\
& =\frac{\sum_{p=0}^{P_{Z_{n}}} \sum_{v=0}^{V_{Z_{n}}} \boldsymbol{c}_{p v}, Z_{n} \phi_{p}(s) \varphi_{v}(g)}{\sum_{p=0}^{P_{Z_{n}}} \sum_{v=0}^{V_{Z_{n}}} \tilde{c}_{p v, Z_{n}} \phi_{p}(s) \varphi_{v}(g)}
\end{aligned}
$$

Once these modal parametric macromodels are built, given a fixed set of values for the parameters, they are reduced to univariate frequency-dependent functions. Their sum represents the final rational univariate model of the matrix $Z$. The stability and passivity for the $\boldsymbol{Z}$ matrix model are ensured by imposing these system properties on the univariate models of the modes [27]. Finally, a state space representation and an equivalent SPICE circuit can be realized for the $Z$ matrix. Concerning the first two parametric macromodeling strategies, the parametric macromodeling of per-unit-length parameters results, based on the experience of the authors, to be more accurate and simple to accomplish. It only requires to model the per-unit-length impedance and admittance $\boldsymbol{Z}_{p u l}(s, g)$ and $\boldsymbol{Y}_{\text {pul }}(s, g)$ as functions of the frequency and other design parameters, instead of the entire set of modal impedances. On the other hand, it is needed to have accurate and physically meaningful per-unit-length parameters, otherwise an overfitting may appear due to the attempts at accurately modeling not physical effects. The possible overfitting present in the models of the per-unit-length parameters leads to an overfitting of the $Z$ matrix model, which can be removed using a pole pruning step to carry a model order reduction out.

\section{Parametric macromodeling of impedances $\boldsymbol{Z}(s, g)$}

The parametric macromodeling of the $\boldsymbol{Z}$ matrix, composed of the sum of the modes $\boldsymbol{Z}_{n}(s, g)$, can be another macromodeling strategy.

$$
\begin{aligned}
\boldsymbol{Z}(s, g) & \simeq \tilde{\boldsymbol{Z}}(s, g)=\frac{\boldsymbol{N}_{Z}(s, g)}{D_{Z}(s, g)} \\
& =\frac{\sum_{p=0}^{P_{Z}} \sum_{v=0}^{V_{Z}} \boldsymbol{c}_{p v, Z} \phi_{p}(s) \varphi_{v}(g)}{\sum_{p=0}^{P_{Z}} \sum_{v=0}^{V_{Z}} \tilde{c}_{p v, Z} \phi_{p}(s) \varphi_{v}(g)}
\end{aligned}
$$

The $Z$ matrix contains the dynamics of all modes, thus the complexity of this macromodeling process, in other terms the number of poles required for a good model, increase in comparison with the modal macromodeling. Increasing the number of conductors and ports as well, this macromodeling strategy with common poles might need too much memory and it is not possible sometimes to satisfy such requirement. Experiments show that this macromodeling strategy is not a good option for complex MTLs with a large number of ports.

\section{Mode selection}

The infinite sum in (9) must be truncated in order to obtain a finite rational representation of the multiconductor transmission line. Two different strategies with a bottom-up approach are followed and shown in the following algorithms, to choose the number of modes in the macromodeling process. They are based on the check of the dominant poles [45] of the modal impedances $\boldsymbol{Z}_{n}$ evaluated on the minimum, mean and maximum values of each design parameter range. If for a certain number mode $n$ all checks prove that the dominant poles are out of a defined bandwidth $\xi \omega_{\max }$ (where $\xi>1$ ), the algorithms end and the number of modes is equal to $n-1$. The algorithms are described for the bivariate case, but the full multivariate formulation is similar.

\section{NUMERICAL MODELING}

A. Two-conductor transmission line with frequencyindependent per-unit-length parameters and linear terminations

In the first example, a two-conductor transmission line, shown in Fig. 1, has been considered.

The per-unit-length parameters are $R_{p u l}=39.78 \Omega / \mathrm{m}$, $L_{p u l}=0.5269 \mu \mathrm{H} / \mathrm{m}, G_{p u l}=2.576 \mathrm{mS} / \mathrm{m}$ and $C_{p u l}=$ 


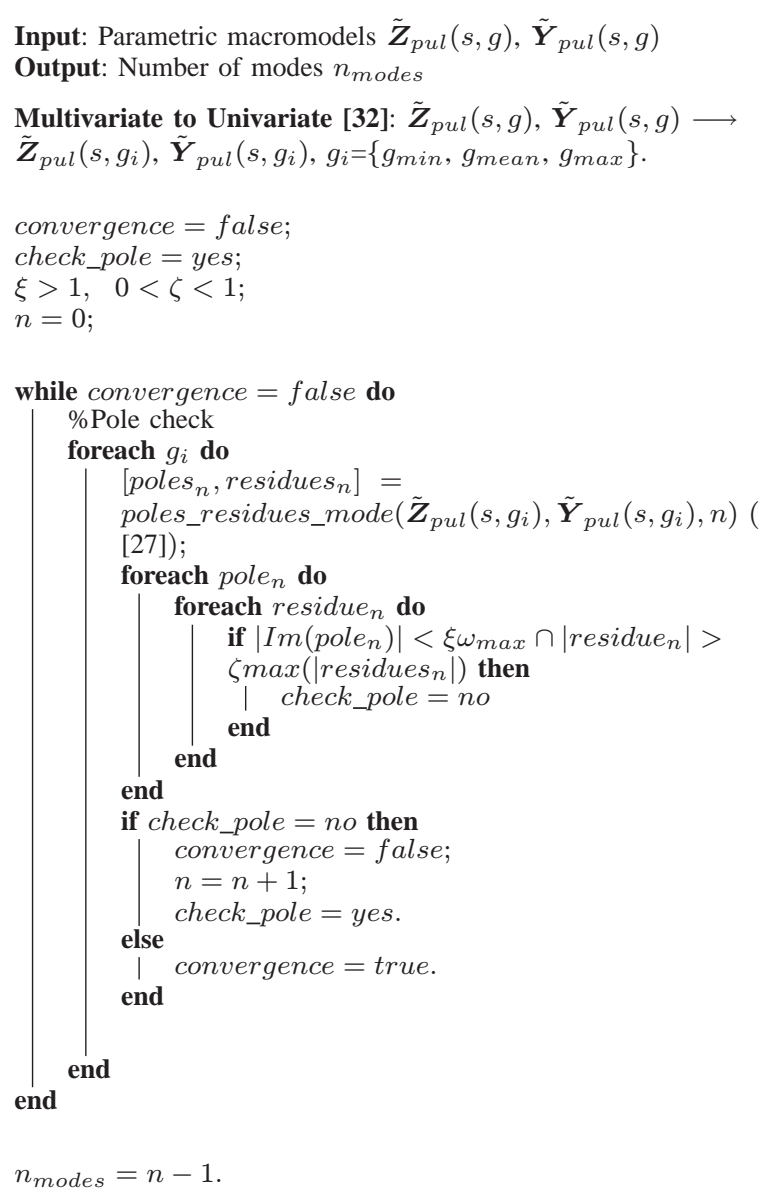

Algorithm 1: Mode selection for the first parametric macromodeling strategy.

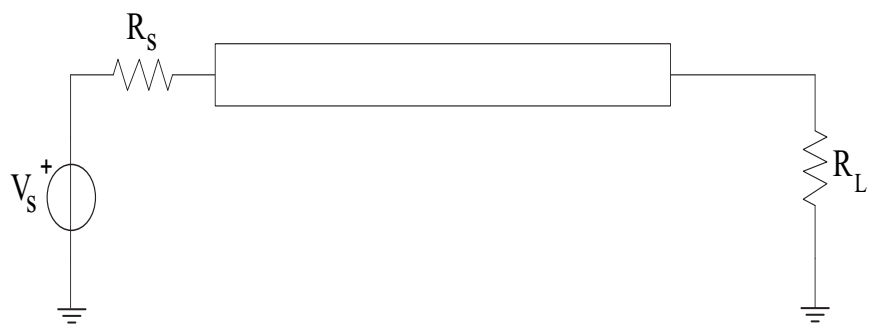

Fig. 1. Two-conductor transmission line with linear terminations.

$50.58 \mathrm{pF} / \mathrm{m}$. The length of the line is considered as parameter in addition to frequency. Their respective ranges are freq $\epsilon$ $\left[100-10^{9}\right] \mathrm{Hz}$ and $\ell \in[1-10] \mathrm{cm}$. In this example the parametric macromodeling of $\boldsymbol{Z}_{p u l}(s, \ell)$ and $\boldsymbol{Y}_{\text {pul }}(s, \ell)$ is not used, because the additional parameter is the length of the line. The infinite series in (9) has been truncated to $n_{\text {modes }}=20$ using the second algorithm for the selection of $n_{\text {modes }}$. All 20 modal impedances have been computed over a reference grid of $251 \times 40$ samples, respectively for frequency and length. We have used $6 \times 6$ samples of the previous grid and 2 poles for both frequency and length, to model all modes. The maximum $R M S_{\text {weighted }}$ error of the parametric macromodels over the reference grid is equal to $7 \cdot 10^{-11}$. The magnitude of the parametric macromodel of $Z_{11}$ is shown in Fig. 2 for

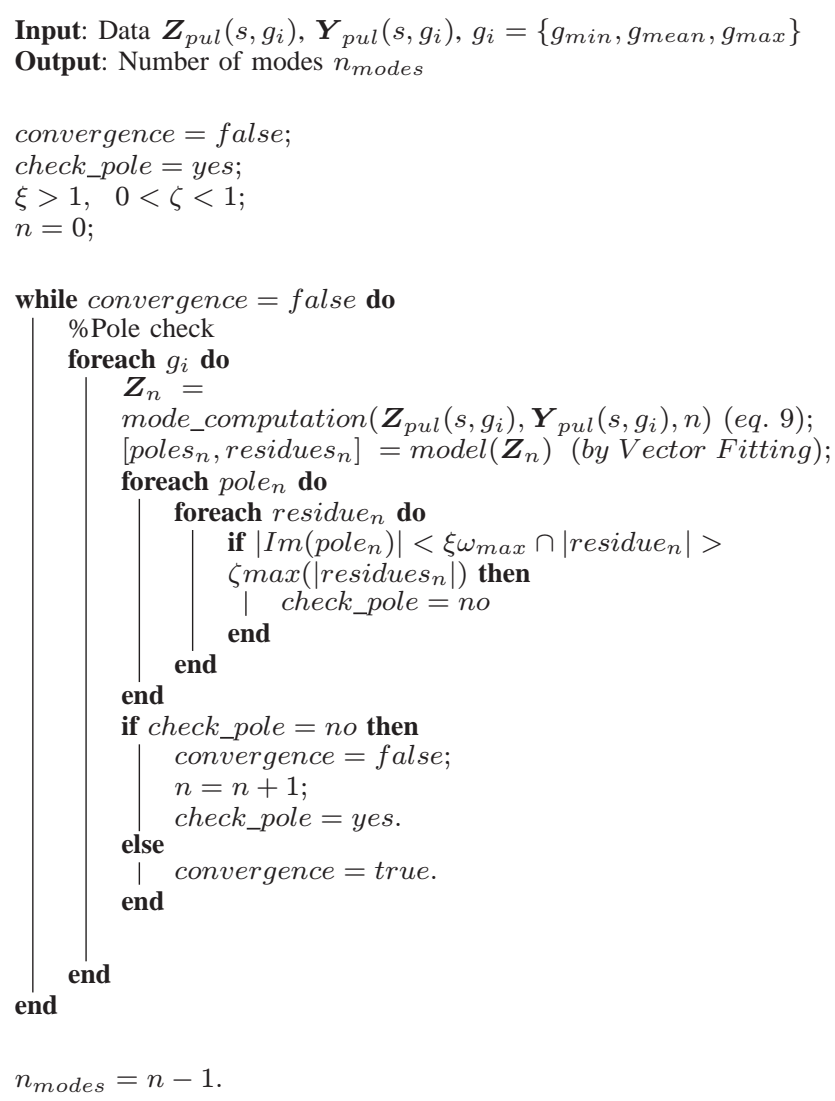

Algorithm 2: Mode selection for the second parametric macromodeling strategy.

TABLE I

DESIGN PARAMETERS OF THE TWO-CONDUCTOR TRANSMISSION LINE STRUCTURE

\begin{tabular}{|c|c|c|}
\hline Parameter & Min & Max \\
\hline Frequency (freq) & $100 \mathrm{~Hz}$ & $10 \mathrm{GHz}$ \\
\hline Length $(\ell)$ & $0.01 \mathrm{~cm}$ & $0.1 \mathrm{~cm}$ \\
\hline
\end{tabular}

modes $n=\{0,1,8\}$. Next, these macromodels have been reduced to univariate frequency-dependent functions for the set of length values $\ell=\{1.46,3.08,4.69,6.31,7.92,9.54\}$ $\mathrm{cm}$. These points have not been used for the generation of the macromodels. The magnitude and the phase of $Z_{12}$ and its univariate model are shown in Figs. 3-4 for modes $n=\{0,1,8\}$ and $\ell=9.54 \mathrm{~cm}$.

The macromodel of the $\boldsymbol{Z}$ matrix, composed of the sum of 20 modes, can be built by using the macromodels of the modal impedances. The $R M S_{\text {weighted }}$ error between the macromodel of the $\boldsymbol{Z}$ matrix and its computation from the exact transmission line theory (TLT) [3] over the reference grid is equal to $2 \cdot 10^{-8}$. The magnitude of the parametric macromodel of $Z_{12}$ is shown in Fig. 5. The comparison is also shown in magnitude and phase for $\ell=6.31 \mathrm{~cm}$ in Figs. 6-7.

The results confirm the high accuracy of the parametric macromodeling strategy in frequency domain. The next step is to show that the accuracy is kept in time-domain as well. The 


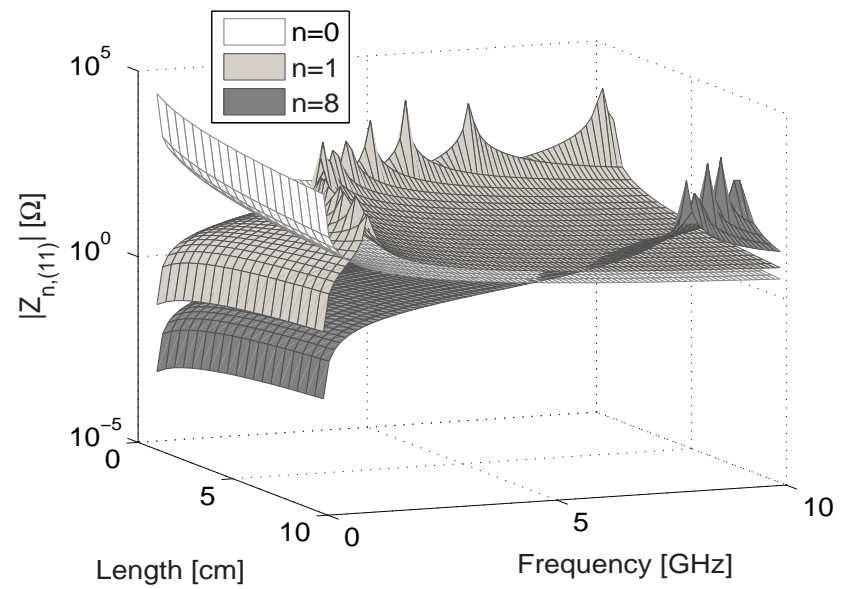

Fig. 2. Magnitude of the parametric macromodel of modal impedance $Z_{n,(11)}(\operatorname{modes} n=\{0,1,8\}$ ).

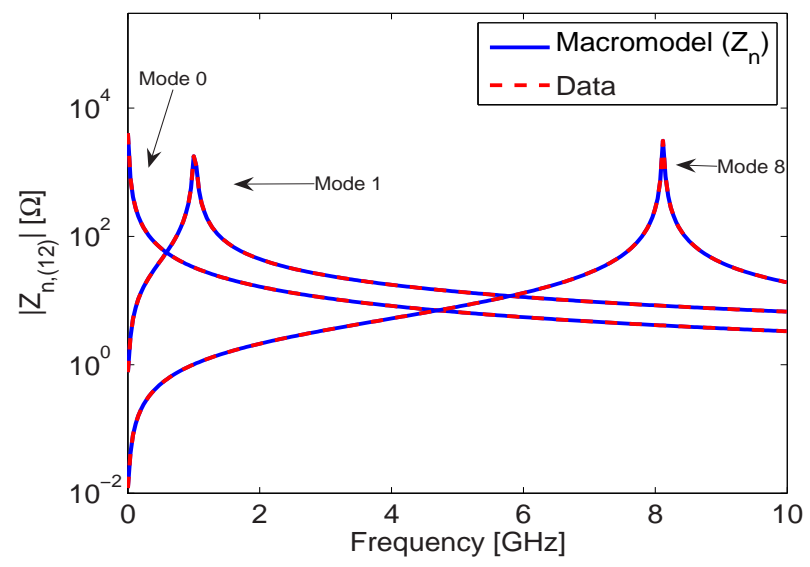

Fig. 3. Magnitude of the macromodel of $Z_{n,(12)}$ (modes $n=\{0,1,8\}$, $\ell=9.54 \mathrm{~cm})$.

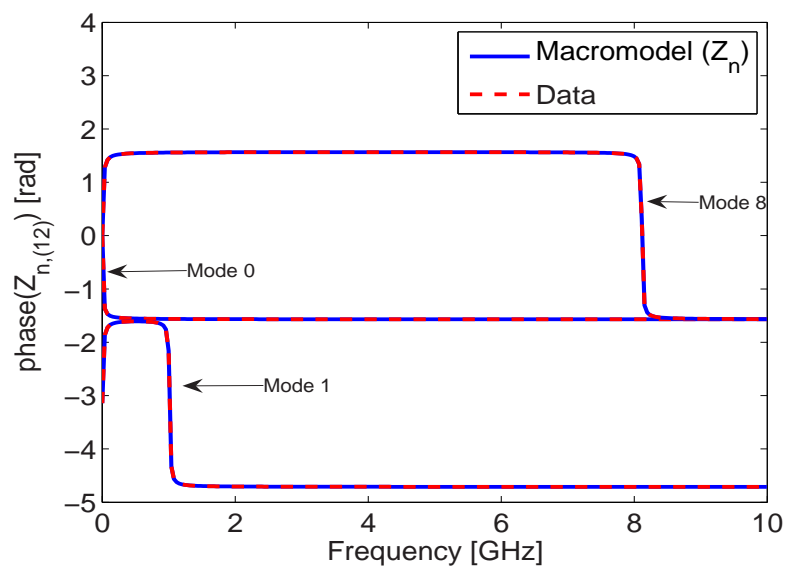

Fig. 4. Phase of the macromodel of $Z_{n,(12)}($ modes $n=\{0,1,8\}, \ell=9.54$ $\mathrm{cm})$.

line has been excited by an impulsive voltage source with amplitude $2 \mathrm{~V}$, rise/fall times $\tau_{r}=\tau_{f}=500 \mathrm{ps}$ and width

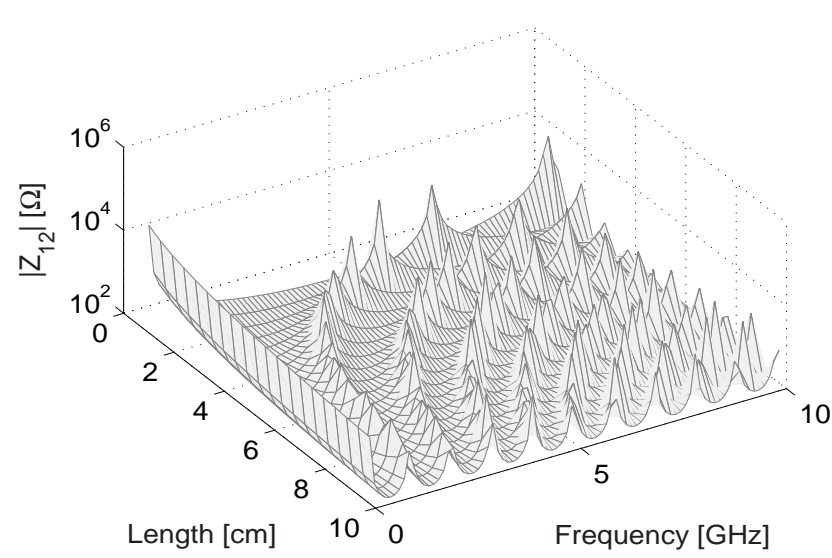

Fig. 5. Magnitude of the parametric macromodel of $Z_{12}$.

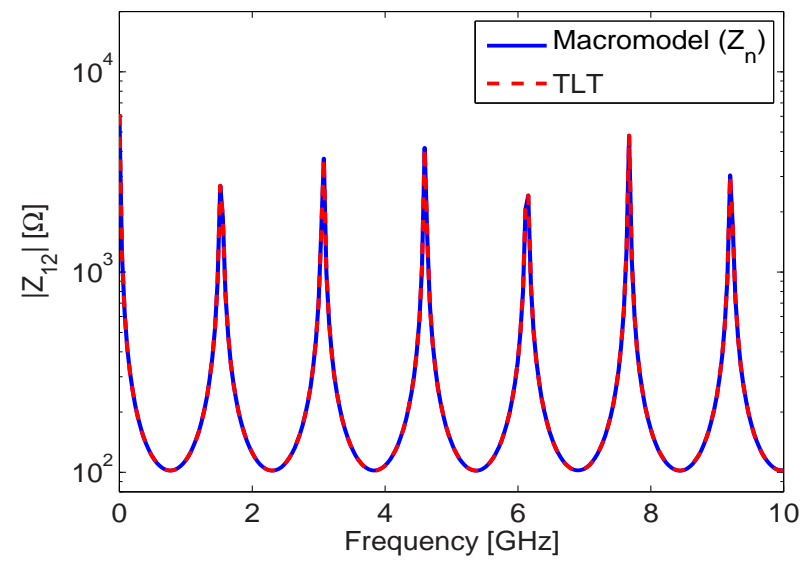

Fig. 6. Magnitude of $Z_{12}(\ell=6.31 \mathrm{~cm})$.

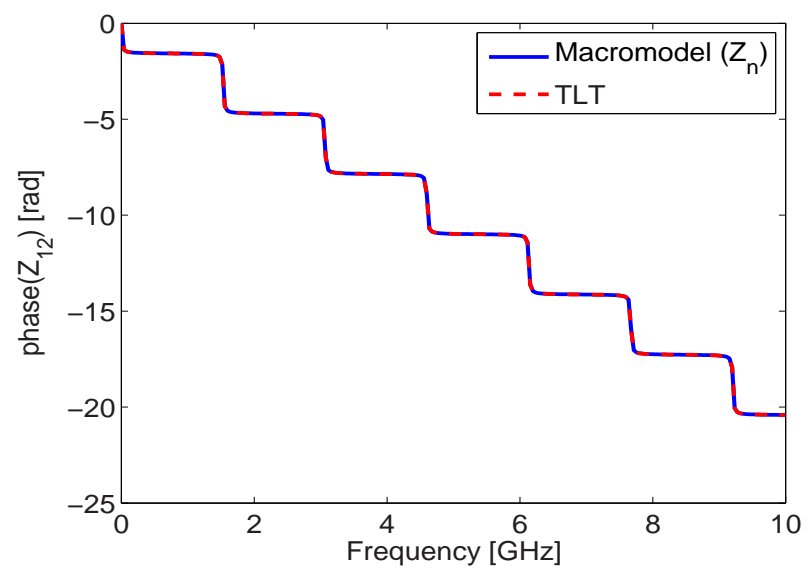

Fig. 7. Phase of $Z_{12}(\ell=6.31 \mathrm{~cm})$.

2 ns. It has been terminated on a driver and load resistance equal to $R_{S}=R_{L}=50 \Omega$. The port voltages have been computed using the exact transmission line theory via Inverse Fast Fourier transform (IFFT) and a state-space realization of the frequency domain macromodel of the $Z$ matrix. The time 
domain results are shown in Figs. 8-9 for the set of length values $\ell=\{1.46,3.08,4.69,6.31,7.92,9.54\} \mathrm{cm}$.

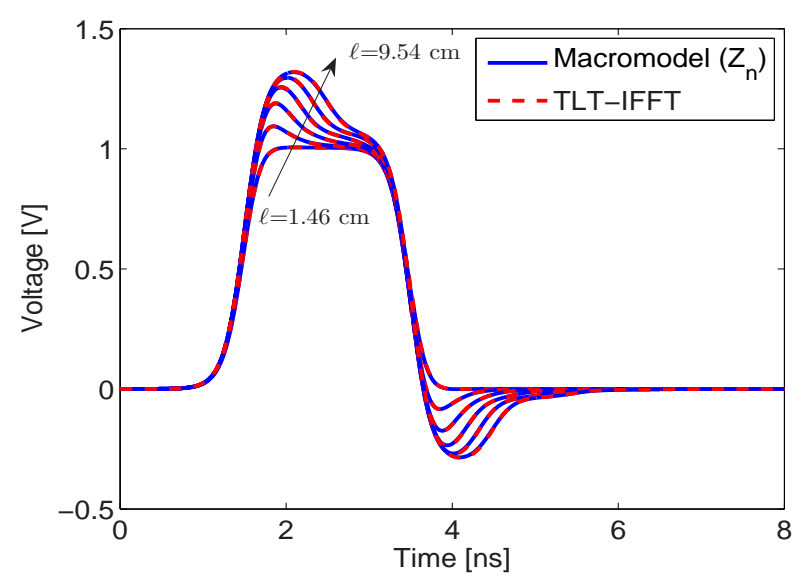

Fig. 8. Input voltage of the line terminated on $R_{S}=50 \Omega$ and $R_{L}=50 \Omega$ $(\ell=\{1.46,3.08,4.69,6.31,7.92,9.54\} \mathrm{cm})$.

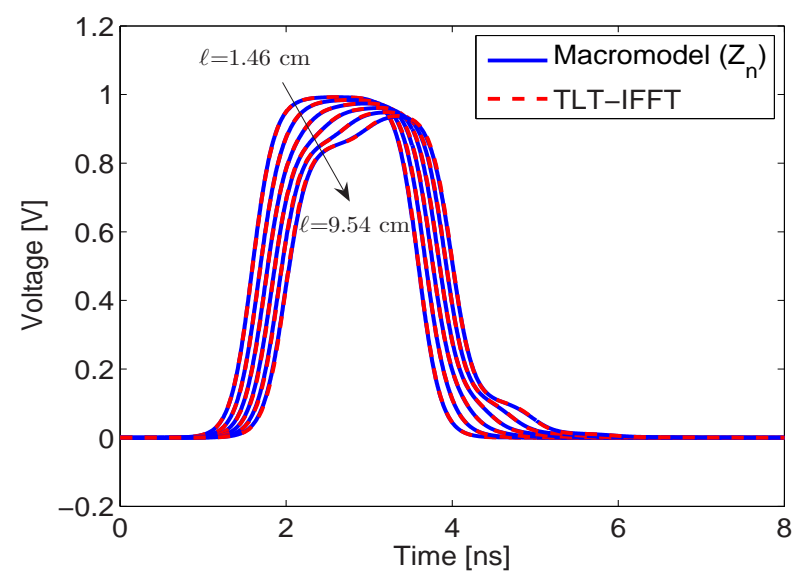

Fig. 9. Output voltage of the line terminated on $R_{S}=50 \Omega$ and $R_{L}=50 \Omega$ $(\ell=\{1.46,3.08,4.69,6.31,7.92,9.54\} \mathrm{cm})$.

As clearly seen, a very good agreement is obtained between the proposed method and the inverse fast Fourier transform, confirming the very high accuracy of the parametric macromodeling strategy in the time-domain as well.

\section{B. Three coupled microstrips with frequency-dependent per- unit-length parameters and linear terminations}

In the second test, a four-conductor transmission line (length $\ell=15 \mathrm{~cm}$ ) with frequency-dependent per-unit-length parameters has been modeled. It consists of three coplanar microstrips over a ground plane. The cross sections is shown in Fig. 10.

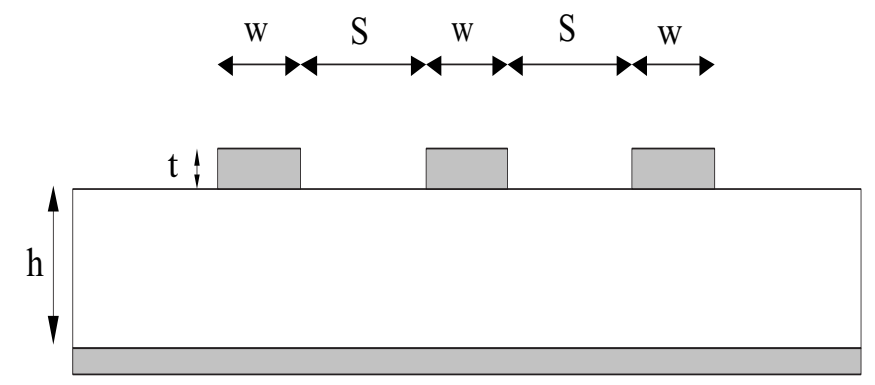

Fig. 10. Cross section of the three coupled microstrips.

The conductors have width $w=100 \mu \mathrm{m}$ and thickness $t=50 \mu \mathrm{m}$. The spacing $S$ between the microstrips is considered as parameter in addition to frequency. The dielectric is $300 \mu \mathrm{m}$ thick and characterized by a dispersive and lossy permittivity which has been modeled by the wideband Debye model [46]. The frequency-dependent per-unit-length parameters have been evaluated using a commercial tool [47]. Both the parametric macromodeling of $\boldsymbol{Z}_{\text {pul }}(s, S), \boldsymbol{Y}_{p u l}(s, S)$ and the modal impedances $\boldsymbol{Z}_{n}(s, S)$ are applied to this example. The infinite series in (9) has been truncated to $n_{\text {modes }}=30$. Both the mode selection tests gave the same result. The ranges of frequency and spacing are freq $\in\left[100-15 \cdot 10^{9}\right] \mathrm{Hz}$ and $S \in[200-400] \mu \mathrm{m}$.

TABLE II

DESIGN PARAMETERS OF THE THREE COUPLED MICROSTRIPS STRUCTURE.

\begin{tabular}{|l|c|c|}
\hline Parameter & Min & Max \\
\hline Frequency (freq) & $100 \mathrm{~Hz}$ & $15 \mathrm{GHz}$ \\
Spacing (S) & $200 \mu \mathrm{m}$ & $400 \mu \mathrm{m}$ \\
\hline
\end{tabular}

First, the parametric macromodeling of $\boldsymbol{Z}_{\text {pul }}(s, S)$ and $\boldsymbol{Y}_{\text {pul }}(s, S)$ is treated. The frequency-dependent per-unitlength parameters have been computed over a reference grid of $251 \times 40$ samples, respectively for frequency and spacing. We have utilized $30 \times 10$ samples of the previous grid and set the number of poles equal to 4 and 2, respectively for frequency and spacing, to model these functions. The maximum $R M S_{\text {weighted }}$ error of the per-unit-length parametric macromodels over the reference grid is equal to $1 \cdot 10^{-5}$. The magnitude of the parametric macromodel of $Z_{p u l,(12)}$ is shown in Fig. 11. 


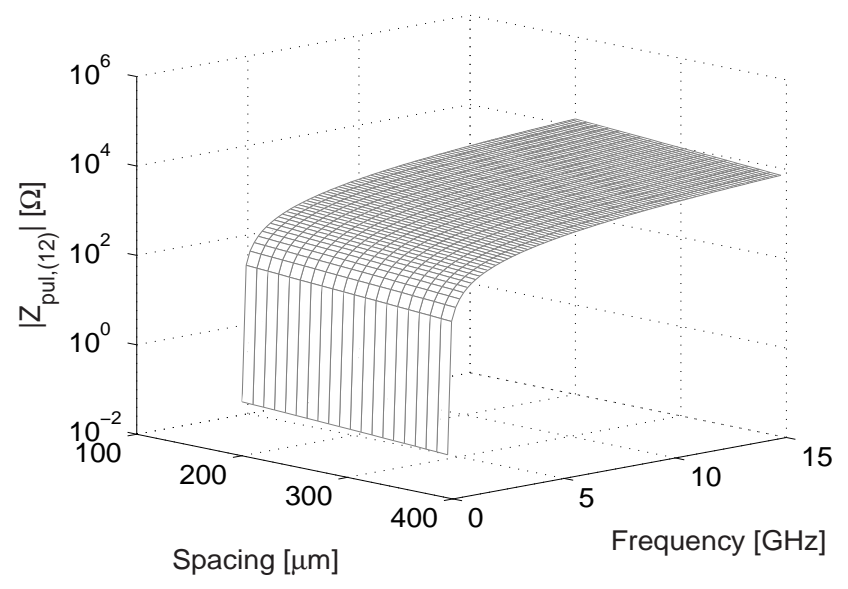

Fig. 11. Magnitude of the parametric macromodel of $Z_{p u l,(12)}$.

Subsequently, these parametric macromodels have been reduced to univariate frequency-dependent functions for the set of spacing values $S=\{231,272,313,354\} \mu \mathrm{m}$. These points have not been used for the generation of the macromodels. Then, the univariate models of the modal impedances have been computed over this spacing points. Concerning the parametric macromodeling of $\boldsymbol{Z}_{n}(s, S)$, all 30 modes have been computed on a grid of $50 \times 15$ samples and these data have been used in the macromodeling process. We have set the number of poles equal to 12 and 2 . Next, the modal parametric macromodels have been reduced to univariate frequencydependent functions for the previous set of spacing values. The maximum $R M S_{\text {weighted }}$ error of all modal parametric macromodels over the reference grid is equal to $3 \cdot 10^{-5}$. The magnitude of the parametric macromodel of $Z_{15}$ built by the second macromodeling strategy is shown in Fig. 12 for modes $n=\{0,1,10\}$. The magnitude and phase of $Z_{11}$ and its respective macromodels are shown in Figs. 13-14 for modes $n=\{0,1,10\}, S=354 \mu \mathrm{m}$.

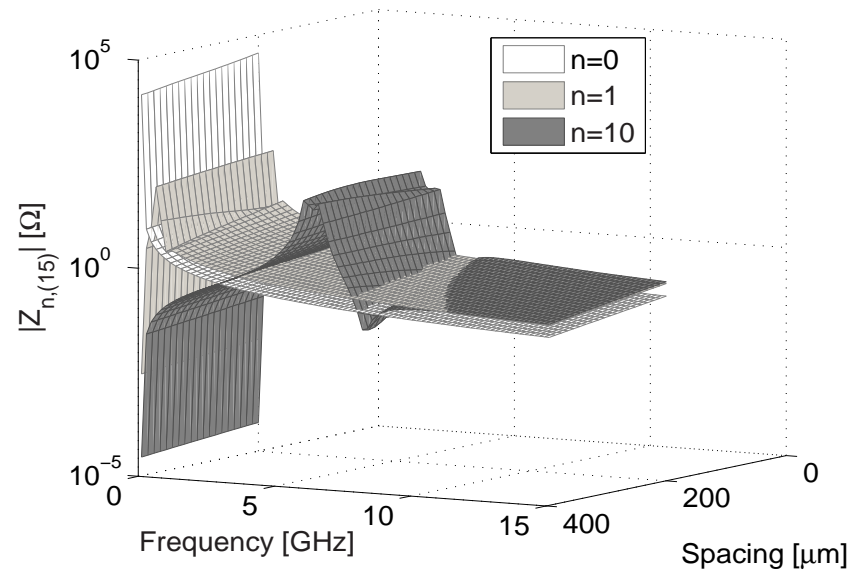

Fig. 12. Magnitude of the parametric macromodel of $Z_{n,(15)}$ by the second strategy (modes $n=\{0,1,10\}$ ).

The macromodel of the $Z$ matrix, composed of the sum of 30 modes, can be built by using the models of modal

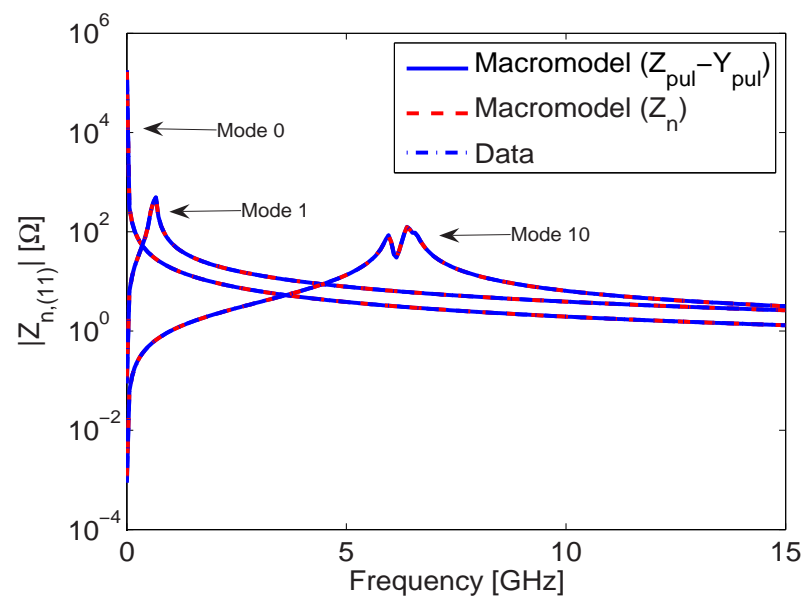

Fig. 13. Magnitude of $Z_{n,(11)}$ (modes $n=\{0,1,10\}, S=354 \mu \mathrm{m}$ ).

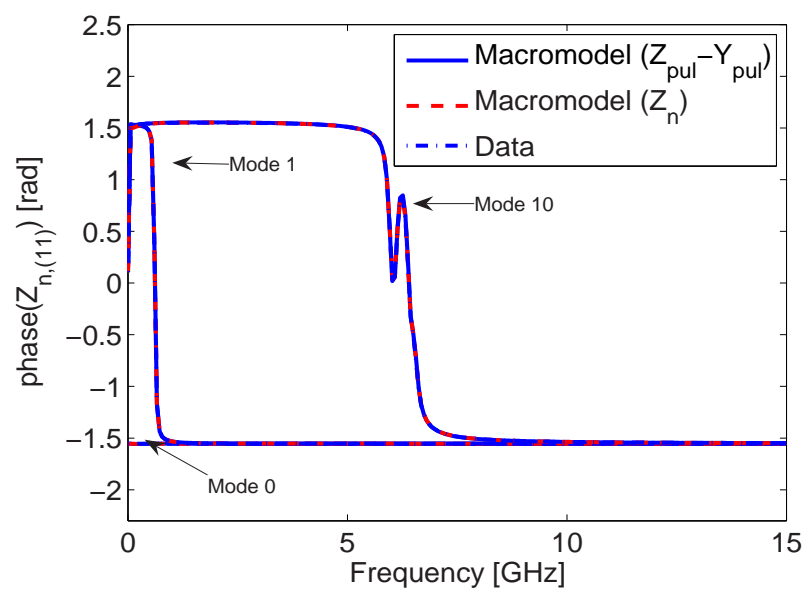

Fig. 14. Phase of $Z_{n,(11)}$ (modes $n=\{0,1,10\}, S=354 \mu \mathrm{m}$ ).

impedances, previously obtained. The $R M S_{\text {weighted }}$ error between the macromodel of the $Z$ matrix and its computation from TLT over the reference grid is equal to $6 \cdot 10^{-4}$ for both the macromodeling strategies. The magnitude of $Z_{14}$ computed by TLT is shown in Fig. 15. The magnitude and the phase of the macromodels of $Z_{14}$ are compared with the results obtained from TLT in Figs. 16-17 for $S=272 \mu \mathrm{m}$.

The results confirm the high accuracy of the parametric macromodeling strategies in frequency domain. As in the previous example, the next step is to show that the accuracy is kept in time-domain as well. The central line has been excited by an impulsive voltage source with amplitude $1 \mathrm{~V}$, rise/fall times $\tau_{r}=\tau_{f}=400 \mathrm{ps}$ and width $80 \mathrm{ps}$. The victim lines have been terminated on the near and far-end by $R_{N E}=50 \Omega$ and $C_{F E}=1 \mathrm{pF}$, while the driven line has been terminated on a driver and load impedance equal to $R_{S}=50 \Omega$ and $C_{L}=1$ $\mathrm{pF}$ (see Fig. 18). The port voltages have been computed using the exact transmission line theory (via-IFFT) and a statespace realization of the frequency domain macromodel of the $Z$ matrix for both the macromodeling strategies. Some time domain results are shown in Figs. 19-20 for the set of spacing 


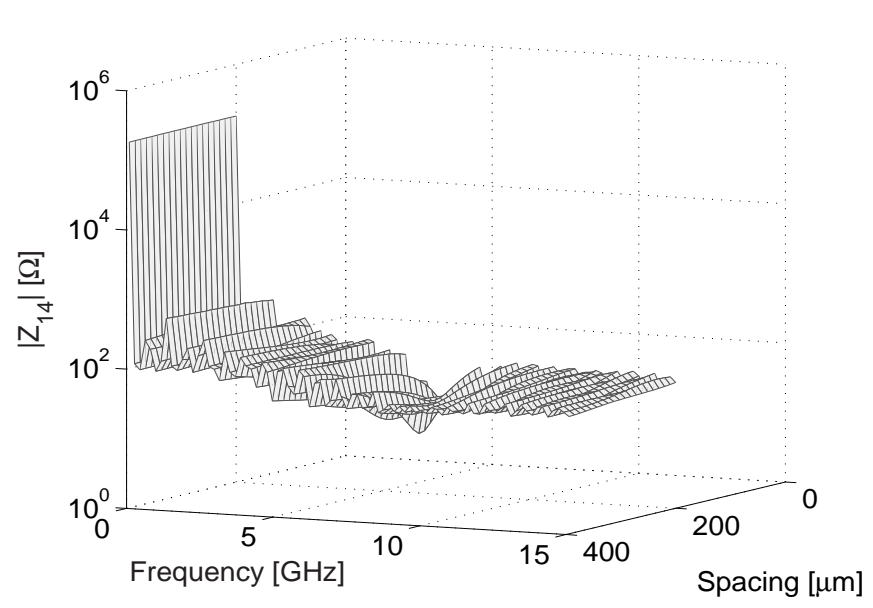

Fig. 15. Magnitude of $Z_{14}$ (TLT).

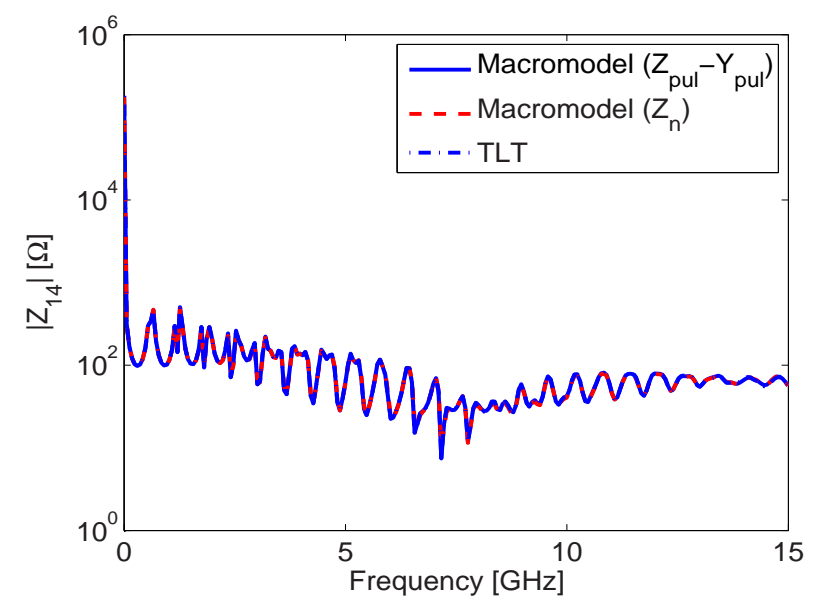

Fig. 16. Magnitude of $Z_{14}(S=272 \mu \mathrm{m})$.

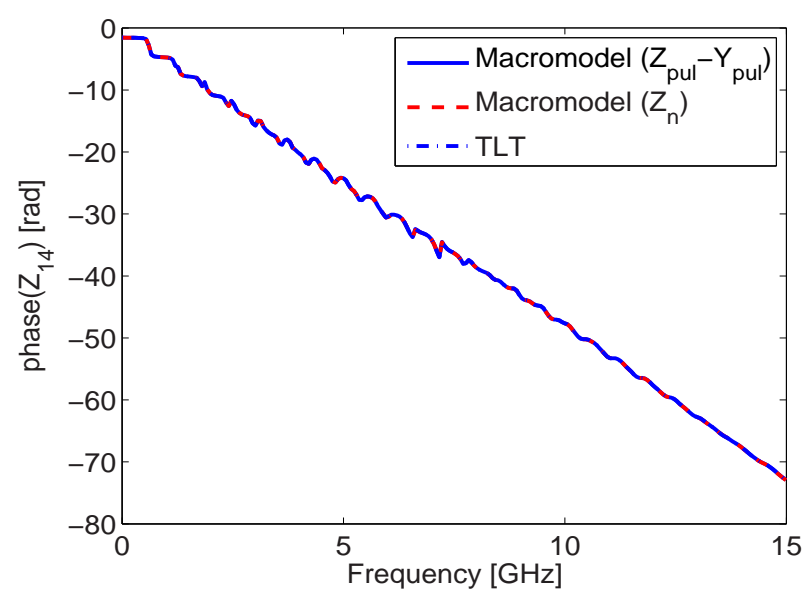

Fig. 17. Phase of $Z_{14}(S=272 \mu \mathrm{m})$.

values $S=\{231,354\} \mu \mathrm{m}$. The port-voltages results confirm that the frequency domain high accuracy of the macromodeling strategies is also preserved in time domain simulations.

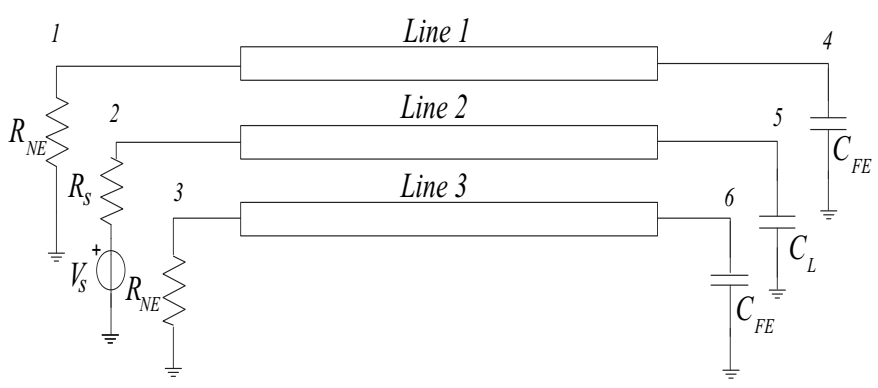

Fig. 18. Three coupled microstrips with linear terminations.

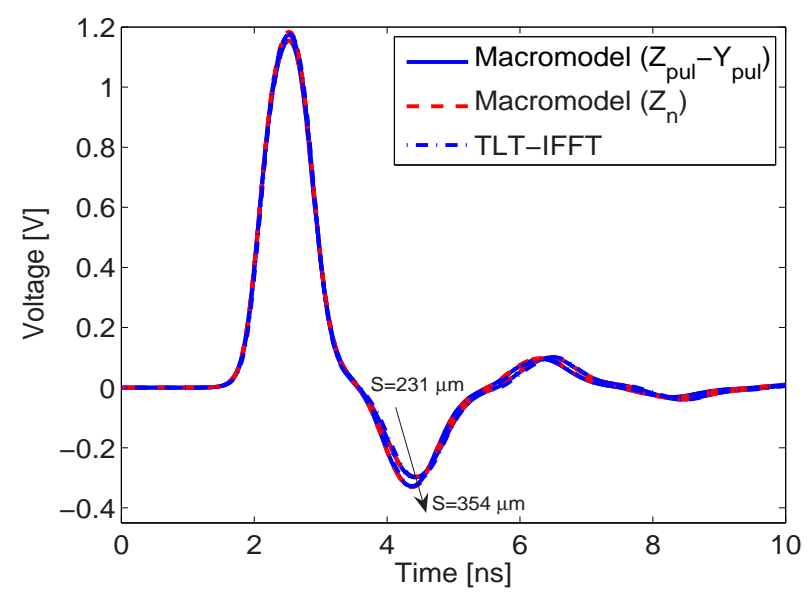

Fig. 19. Output voltages of the driven line terminated on $R_{S}=50 \Omega$ and $C_{L}=1 \mathrm{pF}(S=\{231,354\} \mu \mathrm{m})$.

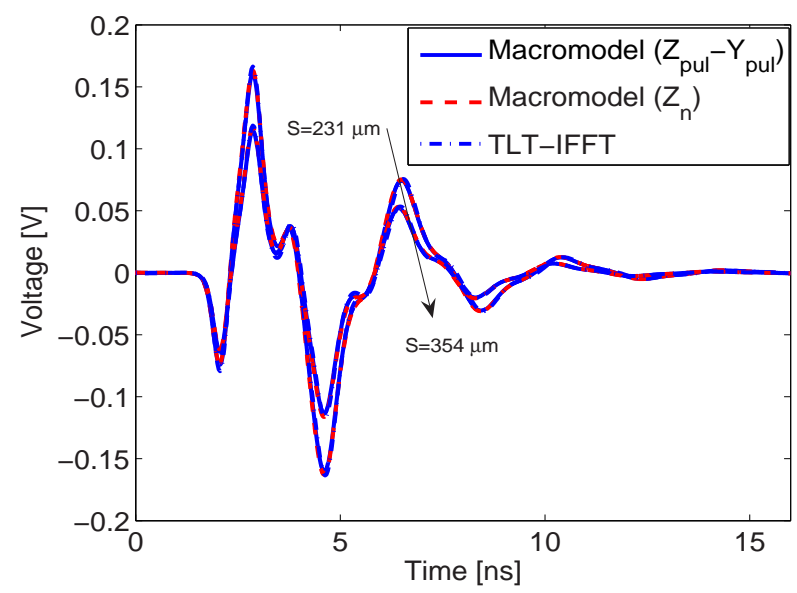

Fig. 20. Output voltages of the victim lines terminated on $R_{N E}=50 \Omega$ and $C_{F E}=1 \mathrm{pF}(S=\{231,354\} \mu \mathrm{m})$.

\section{CONCLUSIONS}

Many second order effects, such as delay, coupling and crosstalk, previously neglected in circuit and system simulations of microwave devices, have become prominent because of increased integration levels and signal speeds. Accurate prediction of these interconnects effects is fundamental for a successful design and requires solution of large systems 
of equations which are often prohibitively CPU expensive. Design space exploration, design optimization and sensitivity analysis are involved in the design framework in addition to regular simulations. Their realization by using full electromagnetic simulations on the entire parameter space is often computationally expensive. Parametric macromodeling techniques that take into account design parameters, such as layout and substrate features, in addition to frequency (or time) are needed to make efficient these design activities. We have presented an innovative parametric macromodeling approach for lossy and dispersive multiconductor transmission lines. It has been found capable to generate accurate rational macromodels with respect to physical and geometrical parameters. The use of the spectral decomposition of the impedance matrix $\boldsymbol{Z}$ leads a significant simplification of the identification process. Two different macromodeling strategies have been investigated. The numerical results have validated the proposed technique and confirmed its accuracy and effectiveness in capturing second order phenomena which are crucial in the analysis and design of high-speed multiconductor transmission lines.

\section{REFERENCES}

[1] A. Deutsch, "Electrical characteristics of interconnects for highperformance systems," Proc. IEEE, invited paper, vol. 86, no. 2, pp. 315-355, 1998

[2] M. Nakhla and R. Achar, Handbook on VLSI. Boca Raton, FL: CRC, 2000.

[3] C. R. Paul, Analysis of Multiconductor Transmission Lines, 2nd ed. New York, NY: John Wiley \& Sons, 2008.

[4] R. Achar, M. Nakhla, "Simulation of high-speed interconnects," Proceedings of the IEEE, vol. 89, no. 5, pp. 693-728, May 2001.

[5] F. Y. Chang, "The generalized method of characteristics for waveform relaxation analysis of lossy coupled transmission lines," IEEE Transactions on Microwave Theory and Techniques, vol. 37, no. 12, pp. 20282038, Dec. 1989.

[6] J. E. Schutt-Ainé and R. Mittra, "Nonlinear transient analysis of coupled transmission lines," IEEE Transactions on Circuits and Systems, vol. 36, no. 7, pp. 959-967, July 1989.

[7] S. Lin and E. S. Kuh, "Transient simulation of lossy interconnects based on recursive convolution formulation," IEEE Transactions on Circuits and Systems, I, vol. 39, no. 11, pp. 879-892, Nov. 1992.

[8] E. Chiprout and M. Nakhla, "Analysis of interconnect network using frequency hopping (CFH)," IEEE Transactions on Computer-Aided Design, vol. 14, no. 2, pp. 186-200, Feb. 1995.

[9] D. B. Kuznetsov, J. E. Schutt-Ainé, "Optimal transient simulation of transmission lines," IEEE Transactions on Circuits and Systems, vol. 43, no. 2, pp. 110-121, Feb. 1996.

[10] M. Celik, A. C. Cangellaris, "Efficient transient simulation of lossy packaging interconnects using moment matching techniques," IEEE Transactions on Comp. Packag. Manufact. Technol. B, vol. 19, pp. 6473, 1996.

[11] M. Celik, A. C. Cangellaris, and A. Yaghmour, "An all-purpose transmission-line model for interconnect simulation in SPICE," IEEE Transactions on Microwave Theory and Techniques, vol. 45, no. 10, pp. 1857-1867, Oct. 1997.

[12] W. T. Beyene and J. E. Schutt-Ainé, "Accurate frequency-domain modeling and efficient circuit simulation of high-speed packaging interconnects," IEEE Transactions on Microwave Theory and Techniques, vol. 45, no. 10, pp. 1141-1147, Oct. 1997.

[13] A. Odabasioglu, M. Celik, and L. T. Pileggi, "PRIMA: passive reducedorder interconnect macromodeling algorithm," IEEE Transactions on Computer-Aided Design, vol. 17, no. 8, pp. 645-654, Aug. 1998.

[14] Q. Yu, J. M. L. Wang and E. S. Kuh, "Passive multipoint moment matching model order reduction algorithm on multiport distributed interconnect networks," IEEE Transactions on Circuits and Systems, I, vol. 46, no. 1, pp. 140-160, Jan. 1999.

[15] A. C. Cangellaris, S. Pasha, J. Prince and M. Celik, "A new discrete time-domain model for passive model order reduction and macromodeling of high-speed interconnections," IEEE Transactions on Comp. Packag. Technol., vol. 22, pp. 356-364, Aug. 1999.
[16] A. Dounavis, X. Li, M. S. Nakhla, R. Achar, "Passive closed-form transmission-line model for general-purpose circuit simulators," IEEE Transactions on Microwave Theory and Techniques, vol. 47, no. 12, pp. 2450-2459, Dec. 1999.

[17] A. Dounavis, E. Gad, R. Achar, M. S. Nakhla, "Passive model reduction of multiport distributed interconnects," IEEE Transactions on Microwave Theory and Techniques, vol. 48, no. 12, pp. 2325-2334, Dec. 2000.

[18] R. Achar, P.K. Gunupudi, M. Nakhla, E. Chiprout, "Passive interconnect reduction algorithm for distributed/measured networks," IEEE Transactions on Circuits and Systems, II, vol. 47, no. 4, pp. 287-301, Apr. 2000.

[19] A. Dounavis, R. Achar, M. Nakhla, "Efficient passive circuit models for distributed networks with frequency-dependent parameters," IEEE Transactions on Advanced Packaging, vol. 23, no. 3, pp. 382-392, Aug. 2000.

[20] — "A general class of passive macromodels for lossy multiconductor transmission lines," IEEE Transactions on Microwave Theory and Techniques, vol. 49, no. 10, pp. 1686-1696, Oct. 2001.

[21] A. Dounavis, R. Achar and M. Nakhla, "Addressing transient errors in passive macromodels of distributed transmission-line networks," IEEE Transactions on Microwave Theory and Techniques, vol. 50, no. 12, pp. 2759-1768, Dec. 2002.

[22] L. F. Knockaert, D. De Zutter, F. Olyslager, E. Laermans, and J. De Geest, "Recovering lossy multiconductor transmission line parameters from impedance or scattering representations," IEEE Transactions on Advanced Packaging, vol. 25, no. 2, pp. 200-205, May 2002.

[23] I. M. Elfadel, H-M. Huang, A. E. Ruehli, A. Dounavis, M. S. Nakhla, "A comparative study of two transient analysis algorithms for lossy transmission lines with frequency-dependent data," IEEE Transactions on Advanced Packaging, vol. 25, no. 2, pp. 143-153, May 2002.

[24] E. Gad and M. Nakhla, "Efficient simulation of nonuniform transmission lines using integrated congruence transform," IEEE Transactions on Very Large Scale Integration (VLSI) Systems, vol. 12, no. 12, pp. 1307-1320, Dec. 2004.

[25] N. M. Nakhla, A. Dounavis, R. Achar and M. S. Nakhla, "DEPACT: delay extraction-based passive compact transmission-line macromodeling algorithm," IEEE Transactions on Advanced Packaging, vol. 28, no. 1, pp. 13-23, Feb. 2005

[26] C. Chen, E. Gad, M. Nakhla and R. Achar, "Analysis of frequencydependent interconnects using integrated congruence transform," IEEE Transactions on Computer-Aided Design, vol. 26, no. 6, pp. 1139-1149, June 2007.

[27] G. Antonini, "A dyadic Green's function based method for the transient analysis of lossy and dispersive multiconductor transmission lines," IEEE Transactions on Microwave Theory and Techniques, vol. 56, no. 4, pp. 880-895, Apr. 2008.

[28] G. W. Hanson and A. B. Yakovlev, Operator Theory for Electromagnetics. Springer, 2002.

[29] P. Gunupudi, R. Khazaka, and M. Nakhla, "Analysis of transmission line circuits using multidimensional model reduction techniques," IEEE Transactions on Advanced Packaging, vol. 25, no. 2, pp. 174-180, May 2002.

[30] P. K. Gunupudi, R. Khazaka, M. S. Nakhla, T. Smy, and D. Celo, "Passive parameterized time-domain macromodels for high-speed transmission-line networks," IEEE Transactions on Microwave Theory and Techniques, vol. 51, no. 12, pp. 2347-2354, 2003.

[31] S. Grivet-Talocia, S. Acquadro, M. Bandinu, F. G. Canavero, I. Kelander, M. Rouvala, "A parameterization scheme for lossy transmission line macromodels with application to high speed interconnects in mobile devices," IEEE Transactions on Electromagnetic Compatibility, vol. 49, no. 1, pp. 18-24, Feb. 2007.

[32] D. Deschrijver, T. Dhaene and D. De Zutter, "Robust parametric macromodeling using multivariate orthonormal vector fitting," IEEE Transactions on Microwave Theory and Techniques, vol. 56, no. 7, pp. 1661-1667, July 2008.

[33] R. Achar, M. Nakhla, "Simulation of high-speed interconnects," Proceedings of the IEEE, vol. 89, no. 5, pp. 693-728, May 2001.

[34] G. Antonini, "Spice equivalent circuits of frequency-domain responses," IEEE Transactions on Electromagnetic Compatibility, vol. 45, no. 3, pp. 502-512, Aug. 2003.

[35] C. R. Paul, Analysis of Multiconductor Transmission Lines. New York, NY: John Wiley \& Sons, 1992.

[36] J. Brandão Faria, Multiconductor Transmission-Line Structures: Modal Analysis Technique. New York, NY: John Wiley \& Sons, 1993.

[37] L. Pillegi, R. Rohrer, C. Visweswariah, Electronic Circuits and System Simulation Methods. McGraw-Hill Book Company, 1995. 
[38] E. C. Levi, "Complex curve fitting," IRE Transactions on Automatic Control, vol. AC-4, pp. 37-44, May 1959.

[39] C. Sanathanan and J. Koerner, "Transfer function synthesis as a ratio of two complex polynomials," IEEE Transactions on Automatic Control, vol. AC-8, no. 1, pp. 56-58, Jan. 1963.

[40] B. Gustavsen and A. Semlyen, "Rational approximation of frequency domain responses by vector fitting," IEEE Transactions on Power Delivery, vol. 14, no. 3, pp. 1052-1061, July 1999.

[41] D. Deschrijver, B. Haegeman and T. Dhaene, "Orthonormal vector fitting: a robust macromodeling tool for rational approximation of frequency domain responses," IEEE Transactions on Advanced Packaging, vol. 30, no. 2, pp. 216-225, May 2007.

[42] B. Gustavsen, "Relaxed vector fitting algorithm for rational approximation of frequency domain responses," Proc. in Workshop Signal Propagation on Interconnects, pp. 97-100, May 2006.

[43] — - "Enforcing passivity for admittance matrices approximated by rational functions," IEEE Transactions on Power Delivery, vol. 16, no. 1, pp. 97-104, Feb. 2001.

[44] D. Saraswat, R. Achar and M. S. Nakhla, "Global passivity enforcement algorithm for macromodels of interconnect subnetworks characterized by tabulated data," IEEE Transactions on Very Large Scale Integration (VLSI) Systems, vol. 13, no. 7, pp. 819 - 832, July 2005.

[45] G. Antonini, "A new methodology for the transient analysis of lossy and dispersive multiconductor transmission lines," IEEE Transactions on Microwave Theory and Techniques, vol. 52, no. 9, pp. 2227-2239, Sept. 2004.

[46] A.R.Djordjević, R.M. Biljić, V.D. Likar-Smiljanić, T.K. Sarkar, "Wideband frequency-domain characterization of FR-4 and time-domain causality," IEEE Transactions on Electromagnetic Compatibility, vol. 43, no. 4, pp. 662-667, Nov. 2001

[47] Simbeor, Electromagnetic Simulation Environment with 3D Full-Wave Field Solver for Multi-Layered Circuits, Simberian Inc., Seattle.

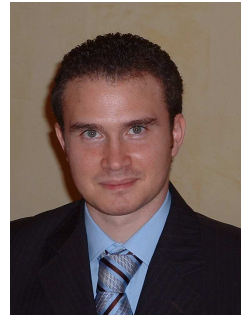

Francesco Ferranti received the B.S. degree in electronic engineering from the Università degli Studi di Palermo, Palermo, Italy, in 2005 and the M.S. degree in electronic engineering from the Università degli Studi dell'Aquila, L'Aquila, Italy, in 2007. Since February 2008, he has been pursuing the Ph.D. in the Department of Information Technology (INTEC) at Ghent University in Belgium. His research interests include robust parametric macromodeling, rational least-squares approximation, system identification and broadband macromodeling
Giulio Antonini (M'94-SM'05) received the Laurea degree (summa cum laude) in electrical engineering from the Università degli Studi dell'Aquila, in 1994, and the Ph.D. degree in electrical engineering from the University of Rome "La Sapienza," in 1998. Since 1998, he has been with the UAq EMC Laboratory, Department of Electrical Engineering, University of L'Aquila, where he is currently Associate Professor. His research interests focus on EMC analysis, numerical modeling, and in the field of signal integrity for high-speed digital systems. He has authored or coauthored more than 180 technical papers and two book chapters. Furthermore, he has given keynote lectures and chaired several special sessions at international conferences. He holds one European patent. Dr. Antonini was the recipient of the IEEE TRANSACTIONS ON ELECTROMAGNETIC COMPATIBILITY Best Paper Award in 1997, the CST University Publication Award in 2004, the IBM Shared University Research Award in 2004, 2005, and 2006. In 2006, he received a Technical Achievement Award from the IEEE EMC Society "for innovative contributions to computational electromagnetic on the Partial Element Equivalent Circuit (PEEC) technique for EMC applications." He also received the IET-SMT Best Paper Award in 2008. He is vice-chairman of the dell'IEEE EMC Italy Chapter, member of the TC-9 committee, and vice-chairman of the TC-10 Committee of the IEEE EMC Society. He serves as member of the editorial board of IET Science, Measurements, and Technology. He serves as reviewer in a number of IEEE journals.

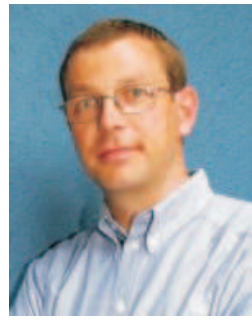

Tom Dhaene was born in Deinze, Belgium, on June 25, 1966. He received the Ph.D. degree in electrotechnical engineering from the University of Ghent, Ghent, Belgium, in 1993. From 1989 to 1993, he was Research Assistant at the University of Ghent, in the Department of Information Technology, where his research focused on different aspects of full-wave electro-magnetic circuit modeling, transient simulation, and time-domain characterization of high-frequency and high-speed interconnections. In 1993, he joined the EDA company Alphabit (now part of Agilent). He was one of the key developers of the planar EM simulator ADS Momentum. Since September 2000, he has been a Professor in the Department of Mathematics and Computer Science at the University of Antwerp, Antwerp, Belgium. Since October 2007, he is a Full Professor in the Department of Information Technology (INTEC) at Ghent University, Ghent, Belgium. As author or co-author, he has contributed to more than 150 peer-reviewed papers and abstracts in international conference proceedings, journals and books. He is the holder of 3 US patents.

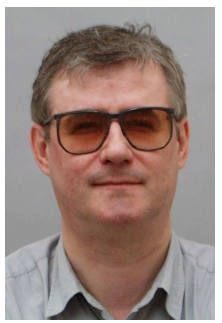

Luc Knockaert received the M. Sc. Degree in physical engineering, the M. Sc. Degree in telecommunications engineering and the $\mathrm{Ph}$. D. Degree in electrical engineering from Ghent University, Belgium, in 1974, 1977 and 1987, respectively. From 1979 to 1984 and from 1988 to 1995 he was working in North-South cooperation and development projects at the Universities of the Democratic Republic of the Congo and Burundi. He is presently affiliated with the Interdisciplinary Institute for BroadBand Technologies (www.ibbt.be) and a professor at the Dept. of Information Technology, Ghent University (www.intec.ugent.be). His current interests are the application of linear algebra and adaptive methods in signal estimation, model order reduction and computational electromagnetics. As author or co-author he has contributed to more than 100 international journal and conference publications. He is a member of MAA, SIAM and a senior member of IEEE. 